TITLE:

\title{
Topological vortex formation in a Bose-Einstein condensate under gravitational field
}

AUTHOR(S):

Kawaguchi, Y; Nakahara, M; Ohmi, T

CITATION:

Kawaguchi, Y ... [et al]. Topological vortex formation in a Bose-Einstein condensate under gravitational field. PHYSICAL REVIEW A 2004, 70(4): 043605.

ISSUE DATE:

2004-10

URL:

http://hdl.handle.net/2433/49949

RIGHT:

Copyright 2004 American Physical Society 
PHYSICAL REVIEW A 70, 043605 (2004)

\title{
Topological vortex formation in a Bose-Einstein condensate under gravitational field
}

\author{
Yuki Kawaguchi, ${ }^{1}$ Mikio Nakahara, ${ }^{2}$ and Tetsuo $\mathrm{Ohmi}^{1}$ \\ ${ }^{1}$ Department of Physics, Kyoto University, Kyoto 606-8502, Japan \\ ${ }^{2}$ Department of Physics, Kinki University, Higashi-Osaka 577-8502, Japan
}

(Received 17 May 2004; published 5 October 2004)

\begin{abstract}
Topological phase imprinting is a unique technique for vortex formation in a Bose-Einstein condensate (BEC) of an alkali-metal gas, in that it does not involve rotation: the BEC is trapped in a quadrupole field with a uniform bias field which is reversed adiabatically leading to vortex formation at the center of the magnetic trap. The scenario has been experimentally verified by Leanhardt et al. employing ${ }^{23} \mathrm{Na}$ atoms. Recently similar experiments have been conducted by Hirotani et al. in which a BEC of ${ }^{87} \mathrm{Rb}$ atoms was used. In the latter experiments the authors found that fine-tuning of the field reverse time $T_{\text {rev }}$ is required to achieve stable vortex formation. Otherwise, they often observed vortex fragmentation or a condensate without a vortex. It is shown in this paper that this behavior can be attributed to the heavy mass of the $\mathrm{Rb}$ atom. The confining potential, which depends on the eigenvalue $m_{B}$ of the hyperfine spin $\boldsymbol{F}$ along the magnetic field, is now shifted by the gravitational field perpendicular to the vortex line. Then the positions of two weak-field-seeking states with $m_{B}=1$ and 2 deviate from each other. This effect is more prominent for BECs with a heavy atomic mass, for which the deviation is greater and, moreover, the Thomas-Fermi radius is smaller. We found, by solving the Gross-Pitaevskii equation numerically, that two condensates interact in a very complicated way leading to fragmentation of vortices, unless $T_{\text {rev }}$ is properly tuned.
\end{abstract}

DOI: 10.1103/PhysRevA.70.043605

PACS number(s): $03.75 . \mathrm{Kk}, 67.57 . \mathrm{Fg}$

\section{INTRODUCTION}

An alkali-metal atom gas undergoes a phase transition to a superfluid phase at very low temperatures $(\sim 0.1 \mu \mathrm{K})$ to form a Bose-Einstein condensate (BEC) [1-4]. This BEC is different from the conventional superfluid ${ }^{4} \mathrm{He}$ in that (i) it is a weakly coupled system for which the Gross-Pitaevskii equation is applicable with high precision at low temperatures; (ii) alkali-metal atoms have hyperfine spin degrees of freedom $\boldsymbol{F}$ and, accordingly, the order parameter has $2 F+1$ components $[5,6]$ while ${ }^{4} \mathrm{He}$ atoms have no such internal structure; (iii) an external magnetic field couples with the hyperfine spin, allowing for easy control of the condensate order parameter, among other things. For example, the atoms ${ }^{23} \mathrm{Na}$ and ${ }^{87} \mathrm{Rb}$ have internal states $F=1$ and $F=2$, for which the number of components is 3 and 5 , respectively.

Associated with the BEC is a quantized vortex, which demonstrates the existence of coherent properties and superfluidity of the condensate. The study of vortices in BECs of alkali-metal atoms started as soon as the BEC was discovered and several scenarios for vortex formation were proposed, namely, (i) dynamical phase imprinting [7,8], (ii) the optical spoon [9-11], (iii) a rotating asymmetric trap [12,13], and (iv) topological phase imprinting [14-18]. We will be concerned in the present paper with vortex formation in the last scenario under the gravitational field.

In topological phase imprinting, the BEC is confined in a quadrupole magnetic field and a bias field orthogonal to it. As the bias field is adiabatically reversed, the condensate spin follows the direction of the local magnetic field and a position dependent order parameter phase is imprinted in the BEC in such a way that a vortex is created along the axis of the trap when the bias field is reversed. This scenario has been verified in beautiful experiments by Leanhard et al.
[18], in which $F=1$ and $F=2$ hyperfine states of ${ }^{23} \mathrm{Na}$ were utilized. An analysis based on Berry's phase [16,19], as well as numerical solutions [15-17], predicts that a vortex thus created has the winding number $2 F$, which was also verified in [18].

In recent experiments conducted by Hirotani et al. [20], where a BEC of ${ }^{87} \mathrm{Rb}$ was used, the authors observed that fine-tuning of the field reverse time $T_{\text {rev }}$ is required for topological formation of a stable vortex. Vortex fragmentation or no vortex was observed for different choices of $T_{\text {rev }}$. We show, in this paper, that the existence of a narrow window in $T_{\text {rev }}$ for successful formation of a stable vortex is attributed to a subtle role played by the gravitational field and the heavier mass of ${ }^{87} \mathrm{Rb}$ compared to ${ }^{23} \mathrm{Na}$.

This paper is organized as follows. In Sec. II, we outline the formalism of the problem based on the multicomponent spinor Gross-Pitaevskii equation. Section III is devoted to the numerical results which verify our statements. It is shown that our $T_{\text {rev }}$ for stable vortex formation approximately reproduces that obtained by Hirotani et al. Conclusions and discussion are given in Sec. IV.

\section{TOPOLOGICAL PHASE IMPRINTING UNDER GRAVITATIONAL FIELD}

We will be mostly concerned with a BEC of ${ }^{87} \mathrm{Rb}$ in the $F=2$ hyperfine state. The case $F=1$ may be analyzed in a similar manner with considerably less effort. We first introduce the order parameter for the $F=2$ BEC and the GrossPitaevskii equation which describes the dynamics of the condensate [21,22]. Topological vortex formation in the presence of a gravitational field is then discussed in detail.

\section{A. Order parameter}

Let us consider a uniform BEC with hyperspin $F=2$. The internal state of the condensate may be classified according 
to the eigenvalue $m_{z}$ of the operator $F_{z}$, where $-2 \leqslant m_{z} \leqslant 2$. The corresponding eigenvector is denoted as $\left|m_{z}\right\rangle: F_{z}\left|m_{z}\right\rangle$ $=m_{z}\left|m_{z}\right\rangle$. As for the eigenvectors we employ the following convention:

$$
\begin{gathered}
|2\rangle=(1,0,0,0,0)^{T}, \quad|1\rangle=(0,1,0,0,0)^{T}, \\
|0\rangle=(0,0,1,0,0)^{T}, \quad|-1\rangle=(0,0,0,1,0)^{T}, \\
|-2\rangle=(0,0,0,0,1)^{T},
\end{gathered}
$$

where $T$ stands for the transpose operation. An arbitrary order parameter is then expanded as

$$
|\Psi\rangle=\sum_{m_{z}=-2}^{2} \Psi_{m_{z}}\left|m_{z}\right\rangle=\left(\Psi_{2}, \Psi_{1}, \Psi_{0}, \Psi_{-1}, \Psi_{-2}\right)^{T} .
$$

In case the external magnetic field $\boldsymbol{B}$ is position dependent, the classification of the hyperfine state in terms of $m_{z}$ is not appropriate and, instead, the eigenstates of $F_{B} \equiv \boldsymbol{F} \cdot \boldsymbol{B} / B$ must be utilized.

Consider the order parameter

$$
|\Psi\rangle_{0}=f_{0}|2\rangle
$$

which satisfies the eigenvalue equation $F_{z}|\Psi\rangle_{0}=2|\Psi\rangle_{0}$. The corresponding eigenstate with respect to an arbitrary $F_{B}$, $F_{B}|\Psi\rangle=2|\Psi\rangle$, with

$$
\boldsymbol{B}(\mathbf{r})=B\left(\begin{array}{c}
\sin \beta \cos \alpha \\
\sin \beta \sin \alpha \\
\cos \beta
\end{array}\right),
$$

is obtained by rotating $\left|\Psi_{0}\right\rangle$ through Euler angles $\alpha, \beta$, and $\gamma$. Explicit calculation shows that

$$
|\Psi\rangle=\exp \left(-i \alpha F_{z}\right) \exp \left(-i \beta F_{y}\right) \exp \left(-i \gamma F_{z}\right)\left|\Psi_{0}\right\rangle
$$

where the last equality defines $|2\rangle_{B}$. This state is under an attractive force toward a region with smaller $B=|\boldsymbol{B}|$ and hence is called a weak-field-seeking state (WFSS). We also obtain $|1\rangle_{B}$ starting from $|1\rangle$ in Eq. (1), which is also called a weak-field-seeking state. The states $|-1\rangle_{B}$ and $|-2\rangle_{B}$, obtained from $|-1\rangle$ and $|-2\rangle$, respectively, are called strongfield-seeking states while the state $|0\rangle_{B}$ is independent of the magnetic field and will be called the neutral state.

\section{B. Gross-Pitaevskii equation}

The dynamics of the $F=2$ BEC in the limit of zero temperature is given, within the mean field approximation, by the time-dependent Gross-Pitaevskii (GP) equation which is extended so that the spin degrees of freedom are taken into account as [21]

$$
\begin{aligned}
i \hbar \frac{\partial}{\partial t} \Psi_{m}= & {\left[-\frac{\hbar^{2}}{2 M} \nabla^{2}-M g x\right] \Psi_{m}+g_{1}\left|\Psi_{n}\right|^{2} \Psi_{m} } \\
& +g_{2}\left[\Psi_{n}^{\dagger}\left(F_{k}\right)_{n p} \Psi_{p}\right]\left(F_{k}\right)_{m q} \Psi_{q} \\
& +5 g_{3} \Psi_{n}^{\dagger}\langle 2 m 2 n \mid 00\rangle\langle 00 \mid 2 p 2 q\rangle \Psi_{p} \Psi_{q} \\
& +\frac{1}{2} \hbar \omega_{L k}\left(F_{k}\right)_{m n} \Psi_{n},
\end{aligned}
$$

where the summations are over $k=x, y, z$ and $-2 \leqslant n, p, q$ $\leqslant 2$. Here, $M$ is the mass of the atom and the gravitational force is assumed to be along the positive $x$ axis. The Larmor frequency is defined as $\hbar \omega_{L k}=\gamma_{\mu} B_{k}$, where $\gamma_{\mu} \simeq \mu_{B}$ is the gyromagnetic ratio of the atom, $\mu_{B}$ being the Bohr magneton. The interaction parameters $\left\{g_{i}\right\}$ are expressed in terms of the $s$-wave scattering length $a_{F}, F$ being the total hyperfine spin of the two-body scattering state, and are given by [21]

$$
\begin{gathered}
g_{1}=\frac{4 \pi \hbar^{2}}{M} \frac{4 a_{2}+3 a_{4}}{7}, \\
g_{2}=-\frac{4 \pi \hbar^{2}}{M} \frac{a_{2}-a_{4}}{7}, \\
g_{3}=\frac{4 \pi \hbar^{2}}{M}\left(\frac{a_{0}-a_{4}}{5}-\frac{2 a_{2}-2 a_{4}}{7}\right) .
\end{gathered}
$$

The parameters are $a_{0}=4.73 \mathrm{~nm}, a_{2}=5.00 \mathrm{~nm}$, and $a_{4}$ $=5.61 \mathrm{~nm}$ for ${ }^{87} \mathrm{Rb}$ atoms.

\section{Topological phase imprinting}

Suppose a WFSS with $m_{B}=2$ is placed in a Ioffe-Pritchard trap. We consider a condensate uniform along the $z$ axis and confined within the $x y$ plane by the quadrupole magnetic field

$$
\boldsymbol{B}_{\perp}(\boldsymbol{r})=\left(\begin{array}{c}
B_{\perp}(r) \cos (-\phi) \\
B_{\perp}(r) \sin (-\phi) \\
0
\end{array}\right)
$$

where $B_{\perp}(r)$ is, to a good approximation, linear in $r$ within the Thomas-Fermi radius: $B_{\perp}(r)=B^{\prime} r$. Here $(r, \phi, z)$ are the cylindrical coordinates where the axis of the magnetic trap is taken as the $z$ axis. To prevent the condensate from escaping from the trap through Majorana flips, a uniform bias field $\boldsymbol{B}_{z}(t)$ must be applied along the $z$ axis, where we have explicitly written the $t$ dependence of the bias field, which is necessary for topological phase imprinting. The total magnetic field is thus given by 


$$
\boldsymbol{B}(\boldsymbol{r}, t)=\boldsymbol{B}_{\perp}(\boldsymbol{r})+\boldsymbol{B}_{z}(t)=\left(\begin{array}{c}
B_{\perp}(r) \cos (-\phi) \\
B_{\perp}(r) \sin (-\phi) \\
B_{z}(t)
\end{array}\right) .
$$

The condensate would be cylindrically symmetric around the $z$ axis if it were not for the gravitation field. In the presence of the gravitational field with a potential $-M g x$, in contrast, the condensate is not symmetric anymore.

The initial condensate profile is determined by solving the stationary GP equation, in which the $m_{B}=2$ WFSS (5) is substituted. The angles in (5) are now given by

$$
\begin{gathered}
\alpha=-\phi=-\tan ^{-1} \frac{y}{x}, \\
\beta=\tan ^{-1} \frac{B_{\perp}(r)}{B_{z}(t)}=\tan ^{-1}\left(\frac{B^{\prime} \sqrt{x^{2}+y^{2}}}{B_{z}(t)}\right) .
\end{gathered}
$$

It is assumed that the initial state is vortex-free, which forces us to choose $\alpha=-\gamma(=-\phi)$ in Eq. (5). The ground state condensate profile corresponds to the lowest energy eigenvalue of the equation

$$
\begin{aligned}
& {\left[-\frac{\hbar^{2}}{2 M} \nabla^{2}-M g x\right]\left(f_{0} v_{m}\right)+\left(g_{1}+4 g_{2}\right) f_{0}^{3} v_{m}+\hbar \omega_{L} f_{0} v_{m}} \\
& \quad=\mu f_{0} v_{m},
\end{aligned}
$$

where $v_{m}=\langle m \mid 2\rangle_{B}, \nabla^{2}=\partial^{2} / \partial x^{2}+\partial^{2} / \partial y^{2}$, and

$$
\hbar \omega_{L}=\gamma_{\mu} \sqrt{B^{\prime 2}\left(x^{2}+y^{2}\right)+B_{z}(t)^{2}} .
$$

The eigenvalue $\mu$ is identified with the chemical potential. If $\left\{v_{m}\right\}^{\dagger}$ is multiplied from the left and $\Sigma_{m}\left|v_{m}\right|^{2}=1$ and similar identities are employed, we obtain the reduced GP equation for $f_{0}$ :

$$
\begin{aligned}
& -\frac{\hbar^{2}}{2 M}\left[\nabla^{2} f_{0}+\sum_{m} v_{m}^{*}\left\{\left(\nabla^{2} v_{m}\right) f_{0}+2 \nabla v_{m} \cdot \nabla f_{0}\right\}\right]-M g x f_{0} \\
& +\left(g_{1}+4 g_{2}\right) f_{0}^{3}+\hbar \omega_{L} f_{0}=\mu f_{0},
\end{aligned}
$$

The initial condensate profile and the chemical potential $\mu$ are obtained by solving Eq. (12) numerically.

The uniform bias field is then reversed as

$$
B_{z}(t)= \begin{cases}B_{z}(0)\left(1-\frac{2 t}{T_{\mathrm{rev}}}\right), & 0 \leqslant t \leqslant T_{\mathrm{rev}}, \\ -B_{z}(0), & T_{\mathrm{rev}}<t .\end{cases}
$$

It has been shown, in previous work $[16,17]$ without taking gravity into account, that a vortex formation takes place at the center of the condensate when the bias field has been reversed. The bias field disappears at $t \sim T_{\text {rev }} / 2$ and the $m_{B}$ $=2$ condensate transforms into $-2 \leqslant m_{B} \leqslant 1$ components through Majorana flips. The components with $m_{B} \leqslant 0$ are not confined and escape from the trap. The final condensate is then made of a mixture of the $m_{B}=1$ and $m_{B}=2$ WFSSs. It is found from Eq. (5) with $\beta=\pi$ that the vortex in the $m_{B}=2$ component has the winding number 4 while a similar condensate amplitude for $m_{B}=1$ tells us that the vortex in this component has the winding number 3 [17]. It is essential, therefore, to employ the full spinor GP equation (6) to analyze this system.

In the presence of the gravitational field, however, the center of the condensate shifts from the axis of the magnetic trap and, moreover, the minimum of the trapping potential, including gravity, depends on the hyperfine state due to the last term in Eq. (6). To be more concrete, the $m_{B}$ component of the condensate in the Ioffe-Pritchard trap is subject to the external potential

$$
V(x, y, t)=\frac{1}{2} m_{B} \gamma_{\mu} \sqrt{B^{\prime 2}\left(x^{2}+y^{2}\right)+B_{z}(t)^{2}}-M g x .
$$

Note that the center of the quadrupole field is $(0,0)$ while the minimum of the potential is

$$
\left(x_{0}, y_{0}\right) \equiv\left(\frac{2 M g\left|B_{z}(t)\right|}{B^{\prime} \sqrt{B^{\prime 2} m_{B}^{2} \gamma_{\mu}^{2}-4 M^{2} g^{2}}}, 0\right)
$$

where we have assumed that $B^{\prime} m_{B} \gamma_{\mu}>2 M g$, which always holds in actual experiments. Otherwise, there is no stable minimum in the $x$ direction and the condensate cannot be confined. Observe that the deviation $x_{0}$ has the same time dependence as $\left|B_{z}(t)\right|$ and that it is larger for $m_{B}=1$ than for $m_{B}=2$, implying that there is a finite distance between the centers of the $m_{B}=1$ and $m_{B}=2$ condensates. It is important to note also that $x_{0}$ is proportional to the atomic mass $M$, provided that $B^{\prime} m_{B} \gamma_{\mu} \gg 2 M g$, and hence the effect of the gravity on the condensate is more prominent for atoms with heavy mass.

The potential $V(x, y, t)$ is approximated by a displaced harmonic potential when $\left|B_{z}\right| \gg\left|B_{\perp}\right|$, taking the form

$$
\begin{aligned}
V(x, y, t) \simeq & \frac{m_{B} \gamma_{\mu} B^{\prime 2}}{4\left|B_{z}(t)\right|}\left(x^{2}+y^{2}\right)-M g x+\frac{1}{2} m_{B} \gamma_{\mu}\left|B_{z}(t)\right| \\
= & \frac{1}{2} M \omega_{\mathrm{HO}}^{2}(t)\left[\left(x-\frac{g}{\omega_{\mathrm{HO}}^{2}(t)}\right)^{2}+y^{2}\right]-\frac{M g^{2}}{2 \omega_{\mathrm{HO}}^{2}(t)} \\
& +\frac{1}{2} m_{B} \gamma_{\mu}\left|B_{z}(t)\right|,
\end{aligned}
$$

where

$$
\omega_{\mathrm{HO}}^{2}(t)=\frac{m_{B} \gamma_{\mu}}{2 M\left|B_{z}(t)\right|} B^{\prime 2} .
$$

The displacement $x_{0}=g / \omega_{\mathrm{HO}}^{2}(t)$ found from Eq. (16) is in agreement with the result (15) when $B^{\prime} m_{B} \gamma_{\mu} \gg 2 M g$. The frequency $\omega_{\mathrm{HO}} \equiv \omega_{\mathrm{HO}}(0)$ serves as an energy scale of the system. The gravitational potential in Eq. (14) leads to two remarkable effects on vortex formation. First, for an $F=2$ BEC, there are two WFSSs; one $\left(m_{B}=1\right)$ has three units of vorticities while the other $\left(m_{B}=2\right)$ has four units. Their positions deviate due to the difference in $m_{B}$, which makes the interaction between the condensates even more complicated. Secondly, the minimum of the potential shifts from the center of the quadrupole field, where the topological vortex formation takes place. A highly quantized vortex with winding number $|n| \geqslant 2$ is unstable against decay into $|n|$ singly quantized vortices. The lifetime of a highly quantized vortex is 


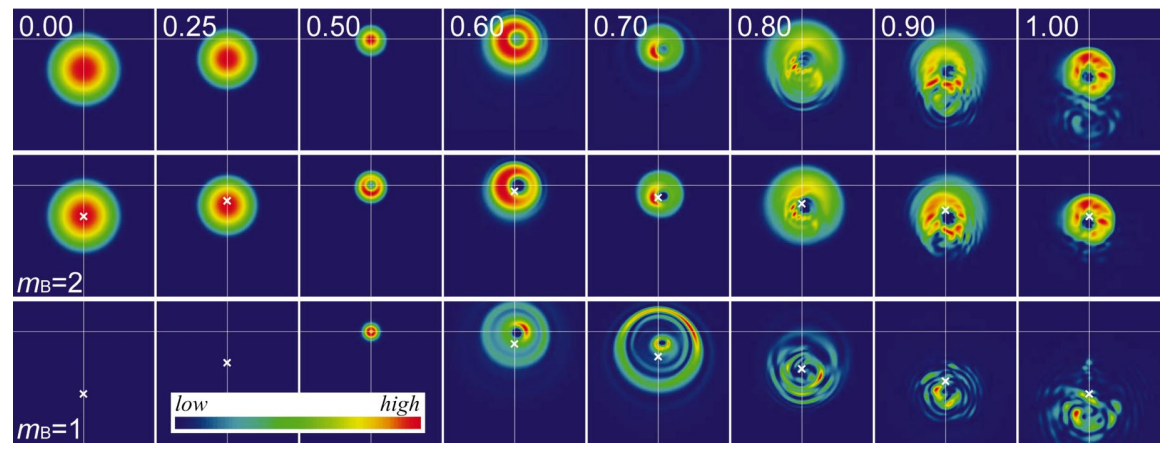

FIG. 1. (Color) Time development of the condensate with $T_{\mathrm{rev}}=4 \mathrm{~ms}$. The white lines show the coordinate system, the origin of which is the center of the quadrupole field where the vortex formation takes place. Upper panel shows the total density of the condensate. The number in the panel shows the normalized time $t / T_{\text {rev }}$. The middle (lower) panel shows the density of the $m_{B}=2\left(m_{B}=1\right)$ component. The cross $(\times)$ shows the minimum of the potential for the respective component. Observe that the center of each $m_{B}$ component follows the minimum of the corresponding potential and accordingly the center of the $m_{B}=1$ component is below that of the $m_{B}=2$ component at $t$ $=T_{\text {rev }}$.

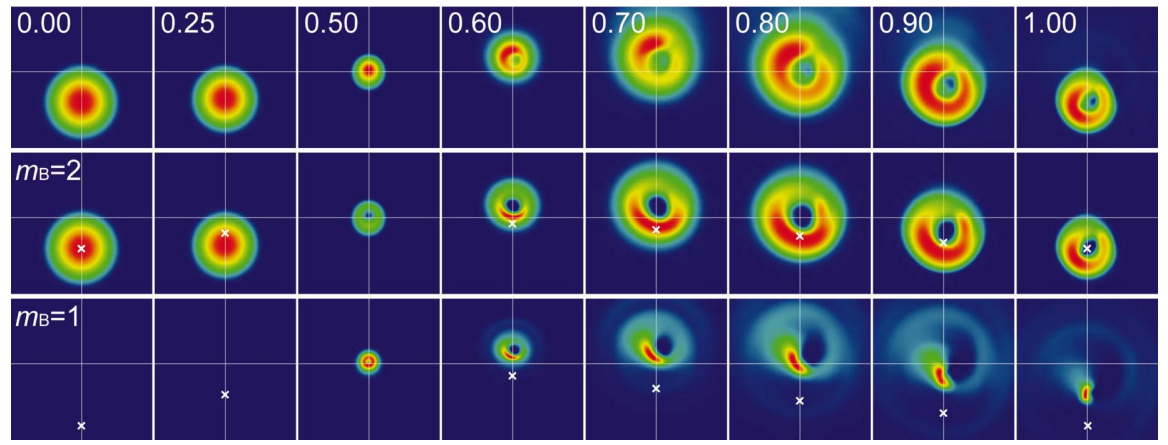

FIG. 2. (Color) Time development of the condensate with $T_{\text {rev }}=2 \mathrm{~ms}$. See the caption of Fig. 1 for the definition of the symbols. The condensate is pushed up while the potential is deformed by reversing $B_{z}$. The $m_{B}=1$ component is still above the minimum of its potential at $t=T_{\text {rev }}$ so that the center of the component is close to that of the $m_{B}=2$ component.

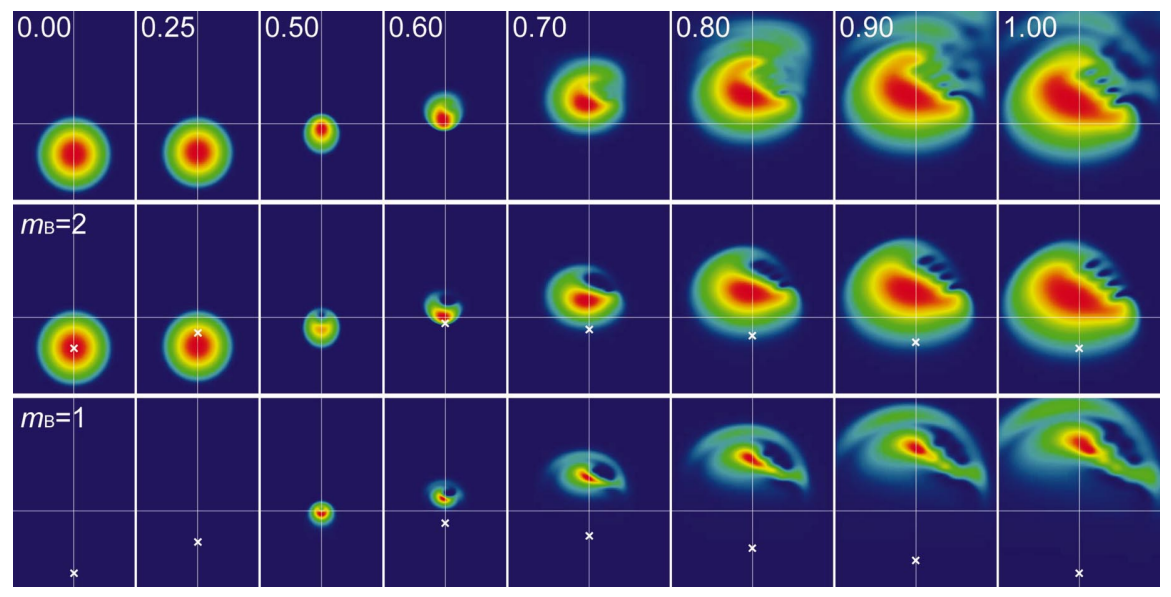

FIG. 3. (Color) Time development of the condensate with $T_{\text {rev }}=1.5 \mathrm{~ms}$. See the caption of Fig. 1 for the definition of the symbols. The condensate is pushed up by a quick deformation of the potential and the centers of both $m_{B}$ components are still above the minima of the potentials at $t=T_{\text {rev }}$. This is more prominent for the $m_{B}=1$ component so that the center of the $m_{B}=1$ component is above that of the $m_{B}$ $=2$ component. 
longer if its axis is at the center of the condensate. If it is off centered, in contrast, it is expected that the vortex moves toward the edge of the condensate and splits into $|n|$ singly quantized vortices. Therefore a topologically created vortex under gravity will be unstable against decay unless some caution is exercised (see the next section). If the deviation of the center of the quadrupole field is large compared to the Thomas-Fermi radius, the "vortex formation" takes place outside the condensate and it is impossible to observe the vortex.

These effects are more prominent in ${ }^{87} \mathrm{Rb}$ compared to ${ }^{23} \mathrm{Na}$. In the experiments of Hirotani et al., the parameter $\omega_{\mathrm{HO}} \simeq 2 \pi \times 330 \mathrm{rad} / \mathrm{s}$ is taken to be the same as in the work of Leanhardt et al. Then the Thomas-Fermi radius $r_{\mathrm{TF}}$ $=a_{\mathrm{HO}} \sqrt{2 \mu / M \omega_{\mathrm{HO}}^{2}}$ has mass dependence $\sim M^{-1 / 2}$ through $a_{\mathrm{HO}}=\sqrt{\hbar / M \omega_{\mathrm{HO}}}$ since the factor $\mu / M$ roughly takes the same value for both experiments.

The above consideration shows that the behavior of the condensate depends heavily on the atomic mass $M$ and the reverse time $T_{\text {rev }}$. We solved the spinor GP equation (6) numerically for various $T_{\text {rev }}$ for both ${ }^{23} \mathrm{Na}$ and ${ }^{87} \mathrm{Rb}$. In the next section, we outline mainly the results for the $F=2$ condensate of ${ }^{87} \mathrm{Rb}$ and then compare them with those for ${ }^{23} \mathrm{Na}$.

\section{NUMERICAL RESULTS}

We have solved the stationary GP equation (12) numerically to obtain the vortex-free initial condensate profile $f_{0}(x, y)$ and the chemical potential $\mu$. We then solved the time-dependent spinor GP equation (6) with $\Psi_{m}=f_{0} v_{m}$ as the initial state. We had to pay special attention to particles escaping form the trap. This is taken care of by introducing an appropriate absorption potential beyond which the condensate amplitude decays exponentially. We found slight interference patters due to reflection at the absorption potential, although we believe that this does not cause any qualitative change in the conclusions.

As for numerical values, we take the following numbers reproducing the experiments of Hirotani et al.: $N$ $=5 \times 10^{5}, \omega_{\mathrm{HO}}=2 \pi \times 330 \mathrm{~Hz}, \omega_{z}=2 \pi \times 17 \mathrm{~Hz}$. The ThomasFermi radius is then $r_{\mathrm{TF}}=3.1 \mu \mathrm{m}$. The initial bias field is $B_{z}(0)=0.5 \mathrm{G}$, which is then reduced to $B_{z}\left(T_{\text {rev }}\right)=-0.5 \mathrm{G}$.

It turns out that a stable vortex formation takes place only for $T_{\text {rev }}$ in a small window around $T_{\text {rev }} \sim 2 \mathrm{~ms}$. The behavior of the BEC is qualitatively different for $T_{\text {rev }}$ out of this window from the case $T_{\text {rev }} \sim 2 \mathrm{~ms}$. This is in harmony with the observation made by Hirotani et al. [20]. Therefore we take $T_{\text {rev }}=4 \mathrm{~ms}, 2 \mathrm{~ms}$, and $1.5 \mathrm{~ms}$ as typical values representing the three classes of $T_{\text {rev }}$.

$$
\text { A. } T_{\text {rev }}=4 \mathrm{~ms}
$$

The change in $B_{z}$ is slow in this case and each WFSS component of the condensate follows its equilibrium position (15) as $B_{z}$ is reversed. There is essentially a single condensate with $m_{B}=2$ at $t<T_{\text {rev }} / 2$. Majorana flips take place at $t$ $\sim T_{\text {rev }} / 2$ in the vicinity of the point where the magnetic field vanishes. The center of the $m_{B}=2$ condensate is separated from that of the $m_{B}=1$ condensate from then on. There exists a vortex with the winding number $4(3)$ in the $m_{B}=2\left(m_{B}\right.$ $=1$ ) condensate, which is created at the center of the quadrapole field at around $t \sim T_{\text {rev }} / 2$. The center of the condensate is at the center of the quadrupole field only at $t=T_{\text {rev }} / 2$ and its subsequent position follows the minimum of the potential, which depends on $m_{B}$. Then these components interact in a complicated manner with each other and vortex fragmentation takes place.

Figure 1 shows the total density profile $\Sigma_{m}\left|\Psi_{m}\right|^{2}$ and the component profile $\left|\Psi_{m_{B}}\right|^{2}$ for $m_{B}=1,2$ with $T_{\text {rev }}=4 \mathrm{~ms}$.

\section{B. $T_{\text {rev }}=2 \mathrm{~ms}$}

A window for stable vortex formation exists in the vicinity of $T_{\text {rev }} \sim 2 \mathrm{~ms}$. The condensate is pushed up by reducing $B_{z}(t)$ and the center of the condensate reaches the center of the quadrupole field at $t \simeq T_{\text {rev }} / 2$ as in the previous case. The minimum of the potential follows the path it took for $0<t<T_{\text {rev }} / 2$ backward after $t=T_{\text {rev }} / 2$. The condensate, however, fails to follow the potential minimum since it has been accelerated upward. During the acceleration, all atoms are in a single hyperfine state and equally accelerated. Following the Majorana flips at $t \simeq T_{\text {rev }} / 2$, each component eventually reaches a peak with some delay, after which it starts to fall toward the corresponding potential minimum. The delay is longer if the acceleration is larger, i.e., $T_{\text {rev }}$ is smaller, and the strength of the confinement is weaker. The latter condition depends on $m_{B}$ as shown explicitly in Eq. (17).

If $T_{\text {rev }}$ is fine-tuned to be around $2 \mathrm{~ms}$, the distance between the two condensates with $m_{B}=1$ and $m_{B}=2$ happens to be small at $t=T_{\text {rev }}$ in spite of the separated potential minima for the respective components. Therefore the $m_{B}=2$ vortex with the winding number 4 is not yet disturbed by the $m_{B}$ $=1$ vortex with the winding number 3 , (see Fig. 2). Observe that the center of the $m_{B}=1$ component is below (above) that of the $m_{B}=2$ component at $t=T_{\text {rev }}$ in Fig. 1 (Fig. 3). Vortex fragmentation occurs at $t>T_{\text {rev }}$ when the distance between the condensates becomes larger as in the previous case.

$$
\text { C. } T_{\text {rev }}=1.5 \mathrm{~ms}
$$

Since the change in $B_{z}(t)$ is fast, the condensate cannot follow the minimum of the potential (15) and does not reach the center of the quadrupole field at $t=T_{\text {rev }} / 2$. The center of the vortex always appears in the center of the quadrupole field and thus the vortex axis appears above the center of the condensate in this case. The vortex thus created has the winding number 4 and a highly quantized off-centered vortex is expected to decay quickly into four singly quantized vortices [23]. This is in fact manifest in Fig. 3 at $t=T_{\text {rev }}$. In the experiments [20], a wavy edge of the condensate is observed when $T_{\text {rev }}$ is smaller compared to that for stable vortex formation. This might be attributed to fragmented vortices.

\section{CONCLUSIONS AND DISCUSSION}

In summary, we have shown that the effect of the gravitational field on the topological vortex formation in BECs of heavy atoms, such as ${ }^{87} \mathrm{Rb}$, is not negligible. Unless the re- 
verse time $T_{\text {rev }}$ is fine-tuned, fragmentation of a multiply quantized vortex takes place during topological phase engineering. This fragmentation is attributed to the existence of two WFSSs with $m_{B}=1$ and 2, whose centers are separated, under gravity, by a distance comparable to the ThomasFermi radius. The WFSS with $m_{B}=1$ supports a vortex with three units of winding number while that with $m_{B}=2$ supports four units.

When the two vortices have the same center, there exists an axially symmetric stable solution [17]. If the vortex centers are displaced from each other, however, the two WFSSs interfere with each other through the $g_{2}$ and $g_{3}$ terms in Eq. (6) so that the deviation from axial symmetry is further amplified, leading to the fragmentation observed in [20].

When the field is reversed so slowly that each hyperfine state follows its potential minimum, the centers of the two hyperfine components deviate from each other as soon as Majorana flips take place at $t \sim T_{\text {rev }} / 2$, leading to vortex fragmentation. When the field is reversed fast, in contrast, the condensate fails to follow the potential minimum and the deviation of two components at $t=T_{\text {rev }}$ is minute. The vortex is, however, imprinted near the edge of the condensate, which leads to splitting of the multiply quantized vortex into singly quantized vortices. The fragmentation pattern often has twofold symmetry, which originates from the vertical displacement of two condensates [23]. To observe a stable multiply quantized vortex, the field reverse time must be taken somewhere between the above two cases.

We have also studied the effect of the gravitational field on the BEC of ${ }^{23} \mathrm{Na}$ with $F=2$, which corresponds to the experiments of Leanhardt et al. We have shown that there exist a similar window of $T_{\text {rev }}$ for topological formation of a stable vortex in this case.

\section{ACKNOWLEDGMENTS}

We are grateful to Tsutomu Yabuzaki, Mitsutaka Kumakura, and Takashi Hirotani for informing us of their experimental results prior to publication. The computation in this work has been done using the facilities of the Supercomputer Center, Institute for Solid State Physics, University of Tokyo. Y.K. and T.O. are supported by a Grant-in-Aid for the 21st Century COE "Center for Diversity and Universality in Physics" from the Ministry of Education, Culture, Sports, Science and Technology (MEXT) of Japan. Y.K. would like to acknowledge support from the Japan Society for Promotion of Science (JSPS) (Project No. 16-0648). M.N. is grateful for partial support of a Grant-in-Aid for Scientific Research from MEXT (Project No. 13135215) and JSPS (Project No. 14540346).
[1] Bose-Einstein Condensation in Atomic Gases, edited by M. Inguscio, S. Stringari, and C. E. Wieman (IOS, Amsterdam, 1999).

[2] Bose-Einstein Condensates and Atom Lasers, edited by S. Martellucci, A. N. Chester, A. Aspect, and M. Inguscio (Kluwer Academic/Plenum, New York, 2000).

[3] C. J. Pethick and H. Smith, Bose-Einstein Condensation in Dilute Gases (Cambridge University Press, Cambridge, U.K., 2002).

[4] L. P. Pitaevskii and S. Stringari, Bose-Einstein Condensation (Oxford University Press, Oxford, 2003).

[5] T. Ohmi and K. Machida, J. Phys. Soc. Jpn. 67, 1822 (1998).

[6] T.-L. Ho, Phys. Rev. Lett. 81, 742 (1998).

[7] J. E. Williams and M. J. Holland, Nature (London) 401, 568 (1999).

[8] M. R. Matthews, B. P. Anderson, P. C. Haljan, D. S. Hall, C. E. Wieman, and E. A. Cornell, Phys. Rev. Lett. 83, 2498 (1999).

[9] K. W. Madison, F. Chevy, W. Wohlleben, and J. Dalibard, Phys. Rev. Lett. 84, 806 (2000).

[10] K. W. Madison, F. Chevy, W. Wohlleben, and J. Dalibard, J. Mod. Opt. 47, 2715 (2000).

[11] J. R. Abo-Shaeer, C. Raman, J. M. Vogels, and W. Ketterle, Science 292, 476 (2001).
[12] E. Hodby, G. Hechenblaikner, S. A. Hopkins, O. M. Maragò, and C. J. Foot, Phys. Rev. Lett. 88, 010405 (2002).

[13] P. C. Haljan, I. Coddington, P. Engels, and E. A. Cornell, Phys. Rev. Lett. 87, 210403 (2001).

[14] M. Nakahara, T. Isoshima, K. Machida, S.-I. Ogawa, and T. Ohmi, Physica B 284-288, 17 (2000).

[15] T. Isoshima, M. Nakahara, T. Ohmi, and K. Machida, Phys. Rev. A 61, 063610 (2000).

[16] S.-I. Ogawa, M. Möttönen, M. Nakahara, T. Ohmi, and H. Shimada, Phys. Rev. A 66, 013617 (2002).

[17] M. Möttönen, N. Matsumoto, M. Nakahara, and T. Ohmi, J. Phys.: Condens. Matter 14, 13481 (2002).

[18] A. E. Leanhardt, A. Görlitz, A. P. Chikkatur, D. Kielpinski, Y. Shin, D. E. Pritchard, and W. Ketterle, Phys. Rev. Lett. 89, 190403 (2002).

[19] M. Berry, Proc. R. Soc. London, Ser. A 392, 45 (1984).

[20] T. Hirotani, T. Fukuhara, A. Kobayashi, M. Kumakura, Y. Takahashi, and T. Yabuzaki (private communication).

[21] C. V. Ciobanu, S.-K. Yip, and T.-L. Ho, Phys. Rev. A 61, 033607 (2000).

[22] M. Koashi and M. Ueda, Phys. Rev. Lett. 84, 1066 (2000).

[23] Y. Kawaguchi and T. Ohmi, Phys. Rev. A (to be published), e-print cond-mat/0402553. 